Marquette University

e-Publications@Marquette

Biomedical Sciences Faculty Research and

Publications

Biomedical Sciences, Department of

$3-2014$

\title{
Nature of the Neurotoxic Membrane Actions of Amyloid- $\beta$ on Hippocampal Neurons in Alzheimer's Disease
}

\author{
Fernando J. Sepúlveda \\ University of Concepción \\ Humberto Fierro \\ University of Concepción \\ Eduardo Fernandez \\ University of Concepción \\ Carolina Castillo \\ University of Concepción \\ Robert W. Peoples \\ Marquette University, robert.peoples@marquette.edu
}

See next page for additional authors

Follow this and additional works at: https://epublications.marquette.edu/biomedsci_fac

Part of the Neurosciences Commons

\section{Recommended Citation}

Sepúlveda, Fernando J.; Fierro, Humberto; Fernandez, Eduardo; Castillo, Carolina; Peoples, Robert W.; Opazo, Carlos; and Aguayo, Luis G., "Nature of the Neurotoxic Membrane Actions of Amyloid- $\beta$ on Hippocampal Neurons in Alzheimer's Disease" (2014). Biomedical Sciences Faculty Research and Publications. 50.

https://epublications.marquette.edu/biomedsci_fac/50 


\section{Authors}

Fernando J. Sepúlveda, Humberto Fierro, Eduardo Fernandez, Carolina Castillo, Robert W. Peoples, Carlos Opazo, and Luis G. Aguayo 
Marquette University

e-Publications@Marquette

\section{Biomedical Sciences Faculty Research and Publications/College of Health Sciences}

This paper is NOT THE PUBLISHED VERSION; but the author's final, peer-reviewed manuscript. The published version may be accessed by following the link in the citation below.

Neurobiology of Aging, Vol. 35, No. 3 (March 2014): 472-481. DOI. This article is (C) Elsevier and permission has been granted for this version to appear in e-Publications@Marquette. Elsevier does not grant permission for this article to be further copied/distributed or hosted elsewhere without the express permission from Elsevier.

\section{Nature of The Neurotoxic Membrane Actions of Amyloid-B On Hippocampal Neurons in Alzheimer's Disease}

Fernando J. Sepúlveda

Laboratory of Neurophysiology, Department of Physiology, University of Concepción, Concepción, Chile Humberto Fierro Laboratory of Neurobiometals, Department of Physiology, University of Concepción, Concepción, Chile Eduardo Fernandez Laboratory of Neurophysiology, Department of Physiology, University of Concepción, Concepción, Chile Carolina Castillo Laboratory of Neurobiometals, Department of Physiology, University of Concepción, Concepción, Chile Robert W. Peoples Laboratory of Biomedical Sciences, Marquette University, Milwaukee, WI Carlos Opazo Laboratory of Neurobiometals, Department of Physiology, University of Concepción, Concepción, Chile 


\title{
Luis G. Aguayo
}

Laboratory of Neurophysiology, Department of Physiology, University of Concepción, Concepción, Chile

\begin{abstract}
The mechanism by which amyloid- $\beta(A \beta)$ produces brain dysfunction in patients with Alzheimer's disease is largely unknown. According to previous studies, $A \beta$ might share perforating properties with gramicidin, a wellaccepted membrane-disrupting peptide. Therefore, we hypothesize that the key steps leading to synaptotoxicity by $A \beta$ and gramicidin involve peptide aggregation, pore formation, and calcium dysregulation. Here, we show that $A \beta$ and gramicidin form aggregates enriched in $\beta$-sheet structures using electron microscopy, and Thioflavin and Congo Red staining techniques. Also, we found that $A \beta$ and gramicidin display fairly similar actions in hippocampal cell membranes, i.e. inducing $\mathrm{Ca}^{2+}$ entry and synaptoxicity characterized by the loss of synaptic proteins and a decrease in neuronal viability. These effects were not observed in a $\mathrm{Ca}^{2+}$ free solution, indicating that both $A \beta$ and gramicidin induce neurotoxicity by a $\mathrm{Ca}^{2+}$-dependent mechanism. Using combined perforated patch clamp and imaging recordings, we found that only $A \beta$ produced a perforation that progressed from a small $\left(\mathrm{Cl}^{-}\right.$-selective pore) to a larger perforation that allowed the entry of fluorescent molecules. Therefore, based on these results, we propose that the perforation at the plasma membrane by $A \beta$ is a dynamic process that is critical in producing neurotoxicity similar to that found in the brains of AD patients.
\end{abstract}

\section{Keywords}

$A \beta$, Alzheimer's disease, Pore formation, Membrane disruption, $A \beta$ toxicity

\section{Introduction}

Alzheimer's disease (AD) is associated with amyloid- $\beta(A \beta)$ peptide aggregate accumulation in the brain parenchyma (Masters et al., 1985). There is strong evidence indicating that $A \beta$ is able to disrupt membranes, causing pore formation (Arispe et al., 1993b). Therefore, $A \beta$ toxicity can be explained in part on the basis of the ion dyshomeostasis induced by $A \beta$, either by its interaction with ligand gated ion channels (NMDA, $\mathrm{Ca}^{2+}$ channels) or by direct lipid disruption (Arispe et al., 1993b, Diaz et al., 2009, Sepulveda et al., 2010). This hypothesis was based on microscopic conductance changes induced by $A \beta$ pore formation in artificial membranes that were highly complex and showed cation selectivity (Arispe et al., 1993a, Kawahara et al., 1997). Single channel measurements in lipid membranes revealed a large variability of single channel conductances (50-500 pS) (Kawahara et al., 1997). Furthermore, it was found that histidine-coordinating molecules that were able to interact with $A \beta$ blocked the ion current flow (Diaz et al., 2009, Sepulveda et al., 2010), suggesting that $A \beta$ amyloid pores can be pharmacologically targeted. Altogether, the data suggest that $A \beta$ does not form a single type of conventional ion channel, but instead contributes to the formation of an array of conducting pores (Kourie et al., 2001). Interestingly, using an "oligomer-enriched" form of $A \beta$, an increase in lipid bilayer conductance was shown (Kayed et al., 2004). However, the latter effect might be related to the solvent action rather than to a specific effect of $A \beta$ (Capone et al., 2009).

With the aim of characterizing functional and structural features that might explain $A \beta$ neurotoxicity, we compared the properties of $A \beta$ and gramicidin, a classical pore-forming peptide. Gramicidin is believed to slowly insert itself into the membrane, allowing the selective passage of cations (Andersen, 1983); and, as an antimicrobial agent, it damages the cell membrane, causing a potent cytotoxic action. Here, we show that both $A \beta$ peptide and gramicidin form $\beta$-sheet structures. Moreover, we found that $A \beta$ and gramicidin aggregates can perforate neuronal membranes and induce neurotoxicity by a similar calcium-dependent mechanism. Unlike $A \beta$, 
gramicidin perforations allowed a reduced entry of fluorescent molecules, suggesting that gramicidin perforates have a smaller inner diameter than $A \beta$ perforates.

\section{Methods}

\subsection{Ethics statement}

All animals used in this study were handled in accordance with the Institutional Animal Welfare Assurance (no. 2008100A) following Comision Nacional de Investigacion Cientifica y Tecnologica de Chile (CONICYT) and National Institutes of Health recommendations.

\subsection{Cell cultures}

Hippocampal neurons were obtained from 18-day Sprague Dawley rat embryos, and human embryonic kidney 293 cells (HEK) were cultured as previously described (Sepulveda et al., 2009). Briefly, the neuronal feeding medium consisted of $90 \%$ minimal essential medium (MEM; BRL Technologies, Rockville, MD), $5 \%$ heatinactivated equine serum, $5 \%$ fetal bovine serum, and a mixture of nutrient supplements. HEK cells were cultivated in Dulbecco's modified Eagle's medium (D-MEM; Life Technologies, Carlsbad, CA) supplemented with $10 \%$ fetal bovine serum (Life Technologies) and streptomycin-penicillin (200 units each, Life Technologies). Cells were maintained with $5 \% \mathrm{CO}_{2}$ at $37^{\circ} \mathrm{C}$.

\section{3. $A \beta$ and gramicidin aggregation}

$A \beta_{1-40}$ ( $r$-Peptide, Bogart, GA), $A \beta_{1-42}$ (Anaspec, Fremont, CA) and gramicidin (G5002, Sigma Aldrich, St. Louis, MO) were prepared according to previous protocols (Akaike, 1996, Sepulveda et al., 2009). Briefly, $A \beta_{1-40}$ and $A \beta_{1-42}$ were dissolved in DMSO $(10 \mathrm{mg} / \mathrm{mL})$ and stored in aliquots at $-20^{\circ} \mathrm{C}$. For the preparation of $A \beta$ aggregates ( $80 \mu \mathrm{mol} / \mathrm{L})$, aliquots of peptide stock ( $250 \mu \mathrm{g}$ in $25 \mu \mathrm{L}$ of DMSO) were added to $700 \mu \mathrm{L}$ of PBS (Gibco, Grand Island, NY), continuously agitated (200 RPM at $37^{\circ} \mathrm{C}$ ) for 90 minutes, and stored at $4{ }^{\circ} \mathrm{C}$. Gramicidin was dissolved in DMSO $(50 \mathrm{mg} / \mathrm{mL})$ and the aggregates were formed after adding PBS to obtain the desired concentration.

\subsection{Electrophysiology recordings}

Patch recordings were performed as previously described (Sepulveda et al., 2009). In short, A $\beta$ and Gramicidin aggregates were used at $500 \mathrm{nmol} / \mathrm{L}$ and $100 \mu \mathrm{g} / \mathrm{mL}$, respectively. Perforated recordings were obtained as follows: the perforating agent was added into the pipette solution and a 5-mV pulse was used to monitor the formation of the perforation at a holding potential of $-60 \mathrm{mV}$ using an Axopatch 200B amplifier (Molecular Devices, Sunnyvale, CA). Data were displayed and stored using a 1322A Digidata acquisition board and analyzed with electrophysiological software (Molecular Devices). The external solution contained the following (in $\mathrm{mmol} / \mathrm{L}$ ): $150 \mathrm{NaCl}, 5.4 \mathrm{KCl}, 2.0 \mathrm{CaCl}_{2}, 1.0 \mathrm{MgCl}_{2}, 10$ glucose, and 10 HEPES (pH 7.4, $330 \mathrm{mOsm}$ ). The standard internal solution in the patch pipette contained the following (in mmol/L): $120 \mathrm{KCl}, 4.0 \mathrm{MgCl}_{2}, 10 \mathrm{BAPTA}$, and 2.0 $\mathrm{Na}_{2}$-ATP (pH 7.4, $\left.310 \mathrm{mOsmol}\right)$. Short applications of GABA $(100 \mu \mathrm{mol} / \mathrm{L})$ were done via lateral motion of a multipipette array ( $200 \mu \mathrm{m}$ in diameter). Some experiments involved an external solution without added calcium, NA7 $(20 \mu \mathrm{mol} / \mathrm{L})$, NA4a $(20 \mu \mathrm{mol} / \mathrm{L})$, or the inactive peptides NA13 and NA15 (20 $\mu \mathrm{mol} / \mathrm{L})$. These peptides were purchased from Peptide 2.0 (Chantilly, VA).

\subsection{Western blots}

The levels of proteins were detected by using the following antibodies: anti-Synapsin I (1:1000; AB1543, Chemicon, Billerica, MA) and anti-SV2 (1:200; Developmental Studies Hybridoma Bank, lowa City, IA). Immunoreactive bands were visualized with ECL plus Western Blotting Detection System (PerkinElmer, Waltham, MA). 


\subsection{Transmission electron microscopy}

Samples of aggregated $A \beta(2.2 \mu \mathrm{g} / \mathrm{mL})$ and gramicidin $(100 \mu \mathrm{g} / \mathrm{mL})$ in phosphate-buffered saline solution (PBS) were placed on nickel grids (Sigma Aldrich, St. Louis, MO) pretreated with 200-mesh Formvar and shaded with carbon. Phosphotungstic acid (0.2\%) was used for contrast and samples were examined under a transmission electron microscope (TEM; JEOL-JEM-1200 EX II, Peabody, MA).

\subsection{Thioflavin and Congo Red staining}

Thioflavin T and Congo Red (Sigma Aldrich, St. Louis, MO) staining were performed as previously described (Klunk et al., 1989, LeVine, 1993). Briefly, the stainings were performed by incubating the suspension of $A \beta$ (2.2 $\mu \mathrm{g} / \mathrm{mL})$ and gramicidin $(100 \mu \mathrm{g} / \mathrm{mL})$ with Congo Red $(10 \mu \mathrm{mol} / \mathrm{L})$, ThS $(200 \mu \mathrm{mol} / \mathrm{L})$, and ThT $(200 \mu \mathrm{mol} / \mathrm{L})$ in PBS with continuous agitation $\left(200 \mathrm{rpm}\right.$ at $37^{\circ} \mathrm{C}$ ) for 30 minutes. The samples were mounted on a coverslip and observed under a fluorescent microscope (Eclipse TE2000; Nikon, Melville, NY, USA) equipped with a CCD camera (Andor Technology, Belfast, UK) and Workbench software 6.0 (Axon Instruments, Molecular Devices, Sunnyvale, CA). The images were processed using ImageJ software (National Institutes of Health, Bethesda, MD).

\subsection{Intracellular calcium imaging}

Neurons were loaded with Fluo-4 AM ( $1 \mu \mathrm{mol} / \mathrm{L}$ in pluronic acid/DMSO, Molecular Probes, Eugene, OR) for 30 minutes at $37^{\circ} \mathrm{C}$. The cells were illuminated (200 milliseconds) under an inverted fluorescent microscope (Eclipse TE; Nikon, Melville, NY, USA), using a computer-controlled Lambda 10-2 filter wheel (Sutter Instruments, Novato, CA). Fluorescent signal from regions of interest (ROI) on different neurons was collected at 1- to 2second intervals during a continuous 5-minute period, using a SensiCam camera (PCO, Kelheim, Germany) associated to Workbench 6 software.

\subsection{Immunocytochemistry}

Hippocampal neurons were fixed for 15 minutes with $4 \%$ paraformaldehyde and permeabilized with $0.1 \%$ triton X-100 in PBS. Monoclonal anti-SV2 (1:200; Developmental Studies Hybridoma Bank, lowa City, IA) and rabbit anti-MAP2 (1:300; Santa Cruz Biotechnology, Dallas, TX CA) were incubated overnight, followed by incubation with a secondary anti-rabbit IgG (1:500; Jackson ImmunoResearch Laboratories, West Grove, PA) conjugated with Cy3 and anti-mouse IgG conjugated with FITC (1:500; Jackson ImmunoResearch Laboratories) for 2 hours.

\subsection{Transfection}

For the NMDAR experiments, human embryonic kidney 293 (HEK293) cells were transfected with NR1-1a, NR2A, and green fluorescent protein ( $p$ GreenLantern plasmid, Invitrogen, Life Technologies, Carlsbad, CA) at a ratio of 2:2:1 using the calcium phosphate transfection kit (Invitrogen, Life Technologies). Expression of green fluorescent protein was used as a marker for positively transfected HEK293 cells and recordings were made after 16 to 20 hours.

\subsection{Neuronal viability}

3-(4,5-Dimethylthiazol-2-yl)-2, 5-diphenyltetrazolium bromide (MTT; Sigma-Aldrich, St. Louis, MO) was added to each well, and the plates were incubated for 6 hours. The Formazan crystals were then dissolved in dimethyl sulfoxide (DMSO; Sigma-Aldrich), giving a spectrophotometrically measurable purple solution. Optical density was read at $570 \mathrm{~nm}$ on the NOVOstar plate reader (BMG Labtech, Ortenberg, Germany).

\subsection{Microscopic current recordings and analysis}

The methodology for single-channel recordings in cell-attached configuration was done using patch pipettes coated with R6101 elastomer (Dow-Corning, Midland, MI) that had a tip resistance of 6 to 10 megaohms after fire polishing. Cells were voltage clamped at $-50 \mathrm{mV}$, and the current was filtered (1-kHz low-pass 8-pole Butterworth) and acquired at 5 to $20 \mathrm{kHz}$ using pClamp software (Molecular Devices). NMDA receptor agonist 
and blockers were applied to cells using a stepper motor-driven rapid solution exchanger (Warner Instrument Corp, Hamden, CT). Cells were maintained in extracellular medium containing the following (in mmol/L): 150 $\mathrm{NaCl}, 5 \mathrm{KCl}, 2 \mathrm{CaCl}_{2}, 10 \mathrm{HEPES}, 10$ glucose; $\mathrm{pH}$ 7.4. The intracellular recording solution contained (in $\mathrm{mmol} / \mathrm{L}$ ): $120 \mathrm{KCl}, 2 \mathrm{MgATP}, 10$ BAPTA, and 10 HEPES, pH 7.2.

\subsection{Simultaneous cell imaging and electrophysiological recordings}

Hippocampal neurons were mounted on an inverted microscope (TE200U, Nikon, Melville, NY, USA) equipped with EMCCD IonX 16-bit camera (Andor, Belfast, UK) plus a patch clamp set-up. The imaging was carried out using a 40X/1.35 objective, and the fluorescent signal for ethidium bromide and a non-hydrolyzable glucose analog (6-NBDG) were obtained using excitation wavelengths of $480 \mathrm{~nm}$ and $545 \mathrm{~nm}$, respectively. The emissions of these fluorescent compounds were collected at $520 \mathrm{~nm}$ and $590 \mathrm{~nm}$, respectively. To diminish the vibration of the stage caused by the movement of the excitation source, a shutter and filter wheel were used to automatically shift the excitation wavelengths. The xenon lamp housing and the filter wheel assembly were coupled to the microscope with a bent optical fiber.

\subsection{Data analysis}

Nonlinear analysis was performed using OriginPro 7.0 (Microcal, Origin Lab, Northhampton, MA). Membrane charge was calculated by integrating the transient capacitative current after subtracting the pipette capacitance; under this condition, the area under the current represented the membrane charge transferred ( $\mathrm{fC}$ ). Moreover, we fitted the current with a standard exponential function using Chebyshev algorithm to calculate the tau for the decay of the current. Using this strategy, we calculated the total charge transferred to the membrane under the patch pipette. All-points amplitude histograms of single channel experiments were generated and fitted with Gaussian functions using ClampFit. The values were expressed as mean \pm standard error of the mean (SEM) from at least 5 to 8 neurons or cells. Statistical differences were determined using 1-way analysis of variance (ANOVA) or paired Student $t$ tests, followed by the Bonferroni post hoc test in some cases.

\section{Results}

\section{1. $A \beta$ and gramicidin aggregate in aqueous solution}

We previously hypothesized that a key step in inducing membrane perforation and synaptotoxicity by $A \beta$ and gramicidin might involve protein aggregation and a subsequent calcium dysregulation (Aguayo et al., 2009). Therefore, we studied the ultrastructural characteristics of $A \beta$ and gramicidin in a saline aqueous solution, and found that $A \beta$ and gramicidin formed fibrillar aggregates (Fig. 1A). To gain further insight into the common features of these structures, we stained them with Thioflavin $\mathrm{T}$ and $\mathrm{S}$, two compounds that react with proteins enriched in $\beta$-sheet structures (LeVine, 1993). Both peptides were positive for these amyloid-staining compounds (Fig. 1B). In addition, both were congophilic and displayed green birefringence under polarized light, which is characteristic for amyloid (Fig. 1C). The presence of $\beta$-sheet structures has been well described for $A \beta$ aggregates (Klunk et al., 1989, LeVine, 1993); however, this is the first time that this structural feature has been associated with gramicidin aggregates. 


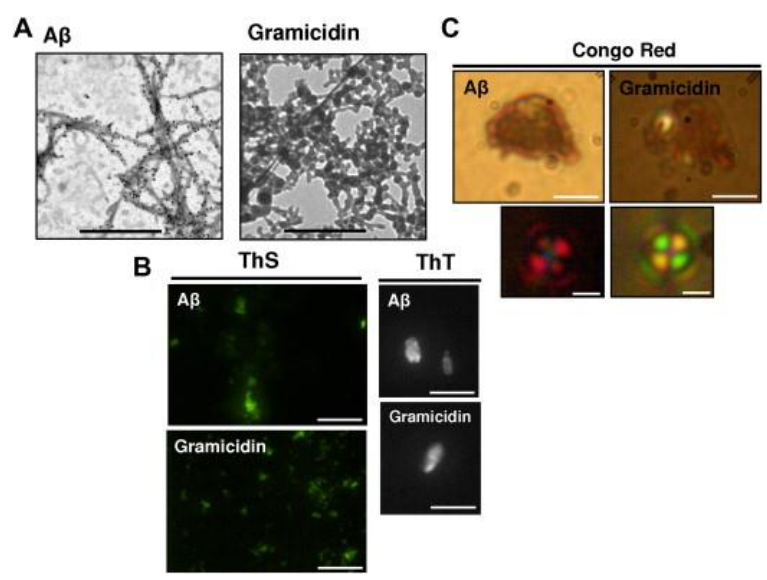

Fig. 1. $A \beta$ and gramicidin form amyloid aggregates. (A) Ultrastructural microphotographs of $A \beta(2.2 \mu \mathrm{g} / \mathrm{mL})$ and gramicidin $(100 \mu \mathrm{g} / \mathrm{mL})$ aggregates in phosphate-buffered saline solution (PBS). A $\beta$ was agitated at $200 \mathrm{RPM}$ at $37^{\circ} \mathrm{C}$ for 90 minutes, and gramicidin was added directly to PBS before observation. (B) Microphotographs show ThS-positive (left panel) and ThT-positive (right panel) $A \beta$ and gramicidin aggregates. The aggregates were obtained as described in A and stained with ThS $(200 \mu \mathrm{mol} / \mathrm{L})$ and ThT $(200 \mu \mathrm{mol} / \mathrm{L})$. (C) A $\beta$ and gramicidin aggregates (obtained under the same conditions as described in A) stained with Congo Red and photographed under polarized light, demonstrating the congophilic-positive nature of both amyloid aggregates. Bars correspond to $500 \mathrm{~nm}(\mathrm{~A}), 10 \mu \mathrm{m}$ (B), $5 \mu \mathrm{m}$ (C, top panels), and $2 \mu \mathrm{m}$ (C, bottom panels).

\section{2. $A \beta$ and gramicidin altered membrane conductance}

To better understand the phenomenon of membrane perforation induced by $A \beta$, we compared the properties of $A \beta$ and gramidicin on neuronal and HEK293 membranes. $A \beta$ and gramicidin aggregates displayed rapid perforating actions (Fig. 2A). In hippocampal neurons, for example, $A \beta$ and gramicidin increased the capacitative current during cell attached recordings (175 \pm 25 and $185 \pm 28 \mathrm{fC}, \mathrm{n}=5-7$ cells). In comparison, the recordings were stable with a control solution in the patch pipette (i.e., 30 minutes) (Fig. 2B). The analysis of charge transferred during the capacitative response and membrane resistance showed that the effect of $A \beta$ and gramicidin on hippocampal neuron and HEK membranes was very similar (Fig. $2 \mathrm{C}$ and $\mathrm{D}$ ). On the other hand, this membrane effect was not produced by the reverse $A \beta$ peptide $\left(A \beta_{40-1}\right)$, a non-aggregating peptide, supporting the idea that $A \beta$ aggregation leads to membrane pore formation, which we previously demonstrated to play a key role in synaptoxicity induced by $A \beta$ (Parodi et al., 2010, Peters et al., 2013, Sepulveda et al., 2010). In addition, the data show that the effects of $A \beta$ on membrane conductance were also blocked by NA7 ( $5 \pm 5 \mathrm{fC}$, $n=5$ ), a mini-peptide that interferes with $A \beta$ perforations, but not by NA13 (135 $\pm 12 \mathrm{fC}$; Fig. 2C and D). The lack of activity found with NA13 is due to the substitution of key His residues by Ala (Diaz et al., 2009, Parodi et al., 2010, Sepulveda et al., 2010). 


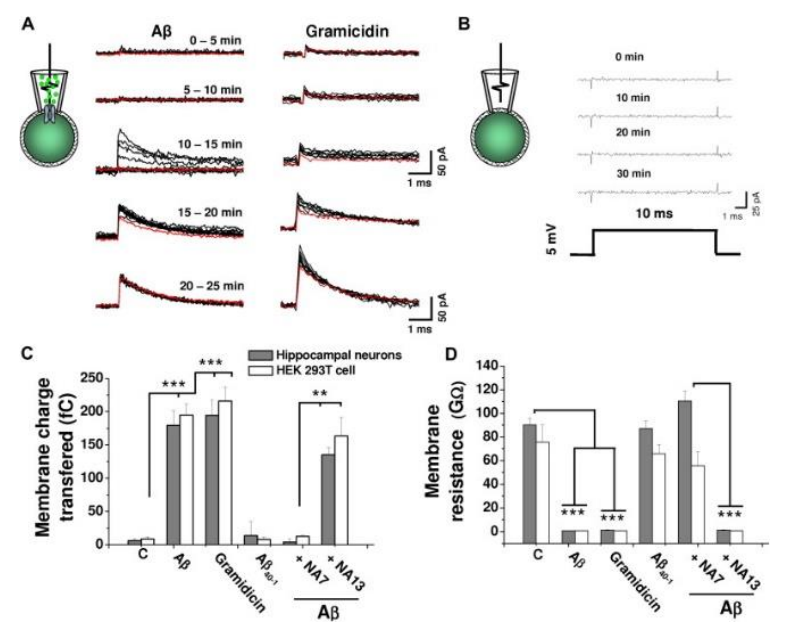

Fig. 2. $A \beta$ and gramicidin caused a time-dependent membrane perforation in hippocampal membranes. (A) Left: effect of $A \beta$ aggregates (500 nanomole/L) on capacitative current. Right: effect of gramicidin ( 50 micromole/L) aggregates on capacitative current. (B) Typical current traces recorded in hippocampal neurons induced by a 5$\mathrm{mV}$ depolarizing pulse recorded with control solution at different time points in the cell-attached mode. ( $\mathrm{C}$ and D) Graphs summarize the effect of $A \beta$ and gramicidin on the transferred membrane charge and membrane resistance, respectively, induced by $5 \mathrm{mV}$ depolarizing pulse. The bars are mean \pm standard error. $\mathrm{n} \geq 5$ cells. ** $p<0.01 ; * * * p<0.005$.

\section{3. $A \beta$ and gramicidin aggregates induce neurotoxicity by a calcium-dependent mechanism}

We previously showed that $A \beta$ was able to decrease the level of a pre-synaptic protein by a $\mathrm{Ca}^{2+}$-dependent mechanism (Parodi et al., 2010, Sepulveda et al., 2010). In the present study, we found that $A \beta$ and gramicidin significantly decreased the levels of SV2 (Fig. 3A, green signal), as shown by a diminished number of puncta (18 \pm 1 vs. $10 \pm 2$ and $9 \pm 1$ puncta/20 $\mu \mathrm{m}$ ) and a decrease in the fluorescence associated with SV2 in hippocampal neurons (Fig. 3B and C). In addition, this synaptotoxic effect of $A \beta$ and gramicidin in hippocampal neurons was confirmed by Western blot analysis (Fig. 3D and E). Interestingly, using fluorescence measurements with Fluo-4, we found that both $A \beta$ and gramicidin increased intracellular calcium in hippocampal neurons (Fig. $4 A$ and $B$ ) at times when they were disrupting the cell membrane (i.e., 15 to 20 minutes) (Fig. 2A). $A \beta$ and gramicidin reduced neuronal viability, measured with an MTT assay (78\% $\pm 3 \%$ and $74 \% \pm 6 \%$ of control at 24 -hour treatments), and these effects were blocked by a reduction in external calcium from 2 to $0.01 \mathrm{mmol} / \mathrm{L}$ (Fig. $4 \mathrm{C}$ and D) supporting a calcium-dependent mechanism. The data also show that culturing hippocampal cells in the absence of external calcium for 24 hours was without effects on viability. Similar results were obtained by adding BAPTA-AM, a calcium chelator (data not shown), supporting the idea that the neurotoxicity induced by $A \beta$ and gramicidin is through a calcium-dependent mechanism. 

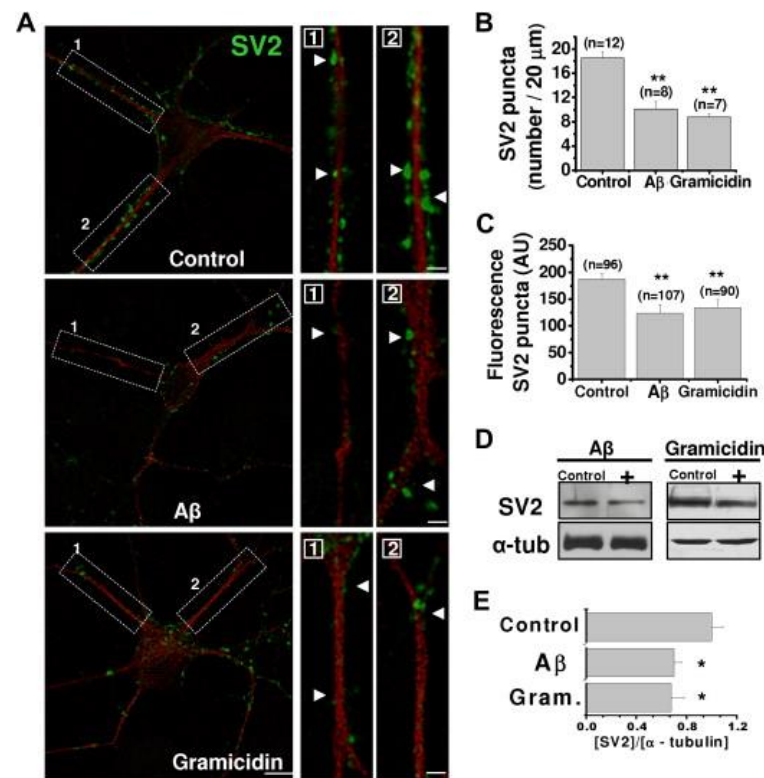

C

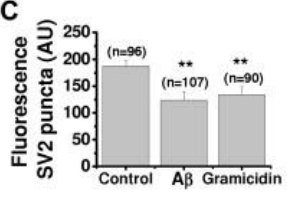

D

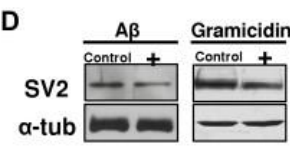

E

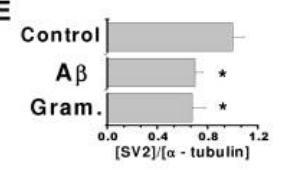

Fig. 3. $A \beta$ and gramicidin induce synaptoxicity. (A) Confocal microphotographs obtained in control and after culturing hippocampal neurons with $A \beta(500 \mathrm{nmol} / \mathrm{L})$ and gramicidin $(50 \mathrm{nmol} / \mathrm{L})$ for 48 hours. Green signal shows SV2 immunoreactivity, and red signal is MAP2, a neuronal marker. Bar scales correspond to $10 \mu \mathrm{m}$ (A, left panels) and $2 \mu \mathrm{m}$ ( $A$, right panels). ( $B$ and C) Graphs illustrate the number of fluorescent SV2 puncta/20- $\mu \mathrm{m}$ length and intensity of the fluorescent signal associated with SV2, respectively, under the same conditions. (D) Western blots show the inhibitory effects of $A \beta$ and gramicidin on SV2 levels measured by chemoluminescence techniques ( $\alpha$-tubulin and $\beta$-actin were used as internal controls). (E) Graphs summarize SV2 levels under the conditions described in $\mathrm{D}$ (from 3 different experiments). ${ }^{*} p<0.05 ;{ }^{*} p<0.01$.

A
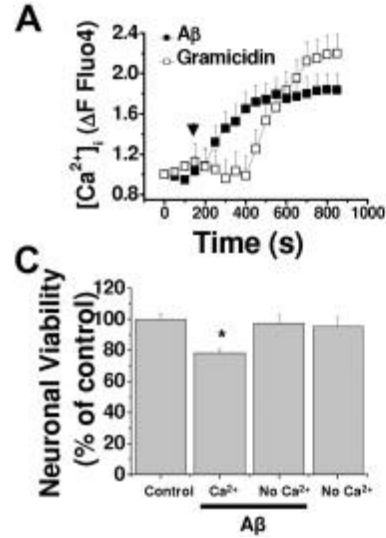

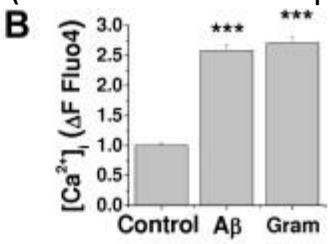

D

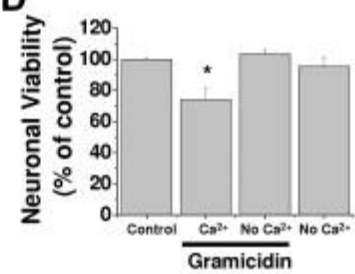

Fig. 4. Toxic effects of $A \beta$ and gramidicin on hippocampal neurons were calcium dependent. (A) Graph shows the effect of exposing hippocampal neurons to $1 \mu \mathrm{mol} / \mathrm{L} A \beta$ (filled symbols) and $1 \mu \mathrm{mol} / \mathrm{L}$ gramicidin (open symbols) on intracellular $\mathrm{Ca}^{2+}$. Arrow indicates the beginning of the application. (B) Effect of $A \beta$ and gramidicin on intracellular $\mathrm{Ca}^{2+}$ levels in hippocampal neurons measured after 20 minutes of application. (C and $D$ ) $A \beta$ and gramidicin neurotoxicity is calcium dependent, as measured by hippocampal neuron viability using an MTT assay after 24 hours of treatment. Data also show that removing extracellular calcium was without effect on viability. Bars are mean \pm standard error. $\mathrm{n} \geq 3$ cells. ${ }^{*} p<0.05 ;{ }^{* *} p<0.01$.

\subsection{Changes in microscopic membrane conductance induced by $A \beta$ and gramicidin}

The effect of a pore-forming peptide to produce the increase in capacitative current results from the sum of small changes in membrane conductances (Ebihara et al., 1995). Therefore, we wanted to detect the channellike microscopic current induced by $A \beta$ and gramidicin during cell-attached recordings to determine the degree of similarities. These experiments were done in HEK cells, because neurons present too many ion channels 
precluding this objective. Using normal internal solution $(140 \mathrm{mmol} / \mathrm{L} \mathrm{CsCl})$, these cells did not display any events reminiscent of single channel currents (not shown). On the other hand, in cells recorded with $A \beta(n=32)$ or gramicidin $(n=13)$, the recording progressed to a perforated configuration, without detectable single channel behavior. Moreover, in some cells, $A \beta$ and gramicidin induced conductance changes that were not like typical single channel behavior (Sepulveda et al., 2010). Fig. 5A, for example, shows representative traces obtained in the presence of $A \beta$ (left) and gramicidin (right) during establishment of perforated conformations. The noisy nature of the microscopic current events produced by both peptides did not allow clear discriminations between open and closed states (Fig. 5B) and plots of all-point current distributions from different patches (A $\beta, n=7$; gramicidin, $n=8$ ) showed multiple levels of peak conductance (100-600 pS), suggesting the occurrence of several membrane disruptions by $A \beta$ and gramicidin (Fig. 5C). Furthermore, it was noteworthy that gramicidin was unable to induce the single channel behavior described in artificial lipid membranes (Andersen, 1983). Under the same conditions, NR1 and NR2A NMDA-induced channels (45 pS) were clearly defined (Fig. 5B, inset).

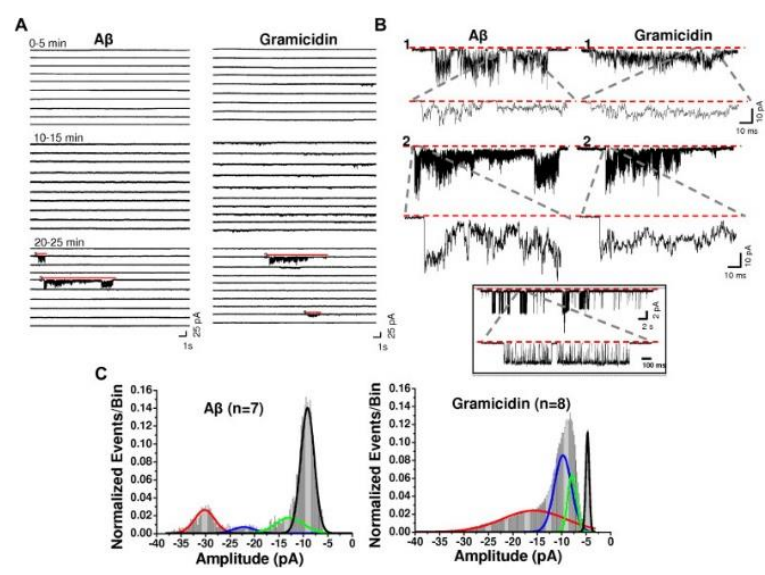

Fig. 5. Study of the microscopic actions of $A \beta$ and gramicidin during membrane perforation. (A) Left: Highsensitivity patch recordings obtained in the presence of $A \beta(500 \mathrm{nM})$ at the indicated times. Right: Microscopic current events showing cell-attached recordings with gramicidin (50 nanomole/L). Note the reduced membrane activity at the times that the peptides were perforating the membrane. (B) Traces are close-ups of representative current traces indicated by numbers in A. (C) Plots show amplitude distribution from the indicated number of membrane patches (colors show representative conductances). (Inset) Classical channel behavior obtained in HEK cells after transfection with NR1-1a and NR2A subunits of NMDA receptors.

\subsection{Reversal potential of $\mathrm{GABA}_{A} \mathrm{RCl}^{-}$current recorded with $A \beta$ and gramicidin cell perforations indicate differential ionic selectivity}

Previous studies showed that the ionophore action of $A \beta$ during the first 15 minutes of pore formation was cation selective, as it behaved similarly to gramicidin, a recognized cation-selective pore-forming peptide (Sepulveda et al., 2010). In the present study, we compared GABA current-voltage (I-V) relationships produced by $A \beta$ and gramicidin with recordings at early (15-20 minutes) and late (30-40 minutes) states of membrane perforation (Fig. 6A). The data show that $A \beta$ (squares), but not gramicidin (circles) produced a membrane perforation that progressed from cation-selective-like to non-selective. At 15 to 20 minutes (filled symbols), for example, the $\mathrm{GABA}_{A}$-activated current reversed at a potential near the $\mathrm{Cl}^{-}$reversal potential $(-40 \mathrm{mV})$ with either $A \beta$ or gramicidin in the pipette, supporting the idea that the perforations allow the passage of cations with short periods of applications. Interestingly, at 30 to 40 minutes of application with $A \beta$ (open squares; see arrows), but not gramicidin (open circles), the $\mathrm{Cl}^{-}$current reversed near $0 \mathrm{mV}$, which is close to the expected value for a non-selective pore, supporting the idea that $A \beta$, but not gramicidin, is able to produce a membrane perforation that changes it selectivity with time. The current traces show that $A \beta$, but not gramicidin, caused a shift of the reversal potential for the $\mathrm{Cl}^{-}$-induced GABA current to $0 \mathrm{mV}$ (Fig. 6B). 

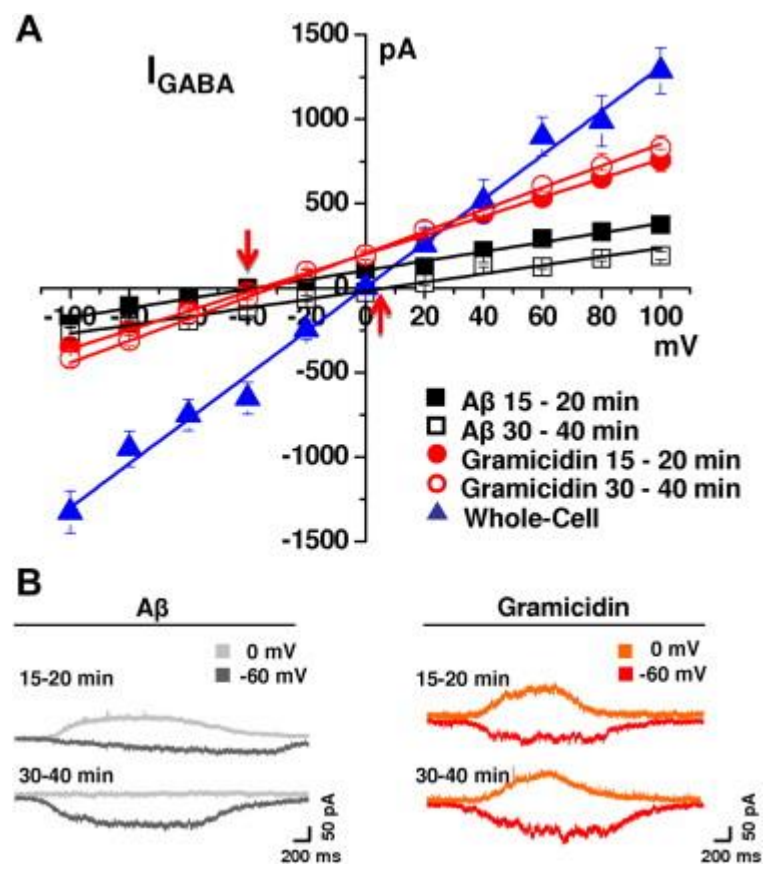

Fig. 6. A $\beta$ pores have a time-dependent transition from cationic-selective to non-selective. (A) Graph shows GABA-induced anionic current-voltage relationships obtained with perforated recordings at 15 and 30 minutes for $A \beta$ (squares) and gramicidin (circles). Symbols represent the mean \pm standard error of the mean from 3 or more neurons. Reversal potentials were close to -50 and $0 \mathrm{mV}$ at 15 to 20 minutes (filled symbols) and 30 to 40 minutes (open symbols) of recording. Note the change on reversal potential for $A \beta$ (red arrows), but not gramicidin, at 30 minutes. (B) Traces show currents activated by $100 \mu \mathrm{mol} / \mathrm{L} \mathrm{GABA}$ at two times of perforated recordings and holding potentials of 0 and $-60 \mathrm{mV}$. Trace recorded at 30 to 40 minutes of $A \beta$-induced perforated recording reverses at $0 \mathrm{mV}$, indicating that the $\mathrm{Cl}^{-}$gradient was lost.

\subsection{A $\beta$ perforations permitted entry of small molecules into hippocampal neurons}

Using a technique that combined perforated patch clamp recordings and cell imaging with a fluorescent nonhydrolyzable glucose analog (6-NBDG, $\sim 1 \mathrm{~nm}$ ) in the patch pipette, we found that small fluorescent probes that have molecular weight and sizes comparable to those of other important cellular metabolites, such as glucose $(\sim 0.9 \mathrm{~nm})$ and nucleotides like ATP $(\sim 1.5 \mathrm{~nm})$, did not pass through intact hippocampal neuron membranes. The control data recorded in the absence of $A \beta$ show that 6-NBDG in the pipette was unable to get into hippocampal neurons (Fig.7A, left). However, disruption of the integrity of the membrane under the patch pipette when the whole-cell configuration was established with a small negative pressure produced the rapid entry of 6-NBDG into the neuron detected within $30 \mathrm{sec}$ (Fig. 7A, right). Thus, the presence of intracellular fluorescence was used as an indicator for membrane leakiness induced by $A \beta$ or gramicidin using 2 small fluorescent molecules with a Van der Waals diameter near $\sim 1 \mathrm{~nm}(6-\mathrm{NBDG}$, em/ex $=490 / 520)$ and $\sim 1.3 \mathrm{~nm}(\mathrm{EtBr}$, em/ex $=545 / 565)$. The data show that $A \beta$-induced perforations allowed the entry of 6-NBDG and EtBr into hippocampal neurons as assessed by the fluorescence increase (Fig. 7C and D), in parallel with the increase in the capacitative current (Fig. $7 E$ and F). The effect of $A \beta$ was dependent on the functionality of the $A \beta$ pores because the fluorescence increase was blocked by NA7 or NA4a, peptides designed to block changes in membrane conductance associated with amyloid perforations (Fig. 7C and D, middle). On the other hand, although gramicidin increased the charge transfer, the pore formed did not produce the entry of $\mathrm{EtBr}$ into the neurons (red squares). The data also show that gramicidin caused a small increase in the fluorescent signal (46 \pm 6 URF) associated with 6-NBDG compared with the effect induced by $A \beta$. In support of the idea that $A \beta$ membrane perforation is a timedependent process, progressing from a small (ion-selective pore) to a large membrane perforation, we found that the time course for the capacitative response, mainly associated with the passage of ions through the 
membrane, increased significantly before uptake of $\mathrm{EtBr}$ and 6-NBDG through $\mathrm{A} \beta$ perforations (Fig. 7E and F), suggesting a late formation of a membrane perforation with a larger pore size than that of gramicidin (Fig. 8).

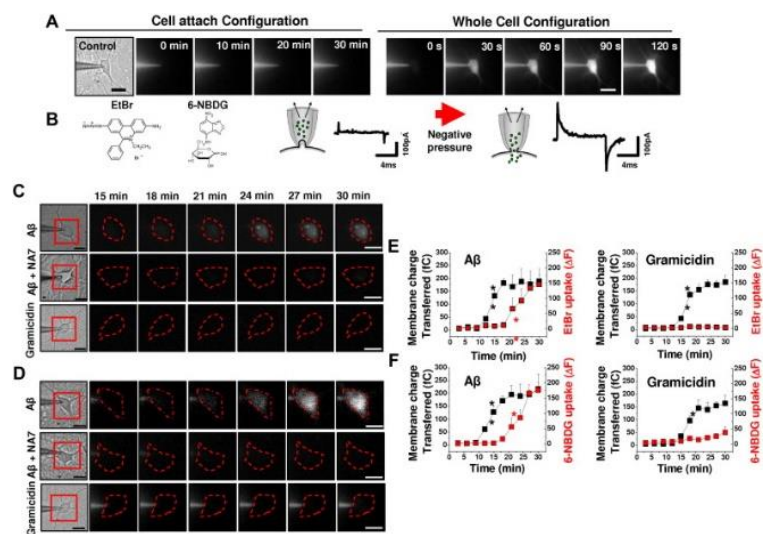

Fig. 7. A $\beta$ perforations allow the entry of small molecules into hippocampal neurons. (A) Left: Cell-attached recording in control conditions using a patch pipette filled with 6-NBDG. Right: Whole-cell configuration allowed rapid entry of 6-NBDG into the neuron. (B) Left: EtBr (diameter $1.3 \mathrm{~nm}$ ) and 6-NBDG (diameter $1 \mathrm{~nm}$ ) 2dimensional structures. Right: Schematic representation of the electrical and fluorescence changes expected under cell-attached and whole-cell configurations. (C) Time-dependent increase in intracellular EtBr fluorescence in the presence of $A \beta$ in the pipette (upper panel) was blocked by the NA7 mini-peptide (middle panel). Gramicidin did not produce the entry of EtBr (lower panel). (D) Time-dependent intracellular increase of 6-NBDG in the presence of $A \beta$ in the pipette (upper panel) was blocked by NA7 mini-peptide (middle panel). Gramicidin allowed a discrete entry of 6-NBDG into hippocampal neurons (lower panel). (E) Graphs show the quantification of membrane charge transferred (capacitative current) and the $\mathrm{EtBr}$ fluorescence induced by $A \beta$ (left) and gramicidin (right) perforations. $A \beta$, but not gramicidin, perforations allowed the entry of EtBr. (F) Graphs show the quantification of membrane charge transferred (capacitative current) and the 6-NBDG fluorescence entry induced by $A \beta$ (left) and gramicidin (right) perforations. ${ }^{*} p<0.05$.

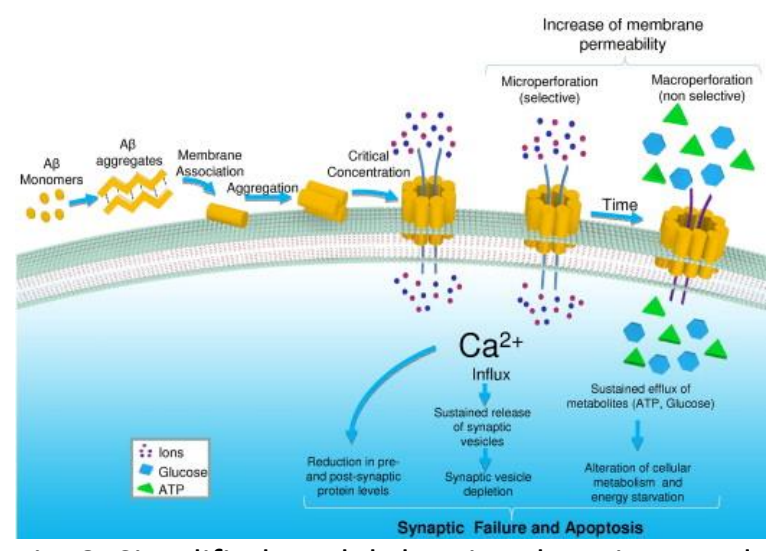

Fig. 8. Simplified model showing the micro- and macro-perforations induced by A . After association with membrane components, the oligomers/aggregates cause disruptions characterized by small ionic pores, allowing cationic influx. Longer exposure of $A \beta$ in the membrane increases the perforation, allowing the entry or exit of large molecules such as EtBr and 6-NBDG. The resulting cellular dyshomeostasis subsequently leads to synaptic failure and apoptosis. 


\section{Discussion}

\subsection{Lack of single channel behavior of $A \beta$ and gramicidin amyloid-like aggregates in native membranes}

It was previously found that gramicidin and $A \beta$ formed pores in artificial lipid membranes having complex single channel conductance (100-1000 pS) and kinetic properties, supporting the idea of complicated molecular behavior (Arispe et al., 1993a, Ebihara et al., 1995). In addition, the channel time course was well described by clear open and closing kinetics for gramicidin (Andersen, 1983) and for $A \beta$ (Kourie, 2001). However, unlike artificial membranes (Andersen, 1983, Arispe et al., 1993a), we found no channel behavior with either ionophore in native membranes. In a few recordings, the response more reminiscent of single channel behavior was an increase in "noise" that we interpreted as a restricted and temporal membrane disruption. The lack of single channel behavior for gramicidin was somewhat surprising, because based on single channel studies in lipid membranes, it was proposed that 2 monomers of gramicidin inserted into the membrane form a functional cationic-selective channel (Kelkar and Chattopadhyay, 2007). In their working model, it was assumed that gramicidin existed only as a monomer in aqueous solution. However, under our working saline conditions, it was evident that gramicidin formed complex and stable oligomeric structures to produce membrane perforations supporting the idea of complex effects on cellular membranes. These structures were of amyloid nature because they reacted with thioflavin ( $T$ and $S$ ) and Congo Red, known to be $\beta$-sheet binding compounds (Klunk et al., 1989, LeVine, 1993). Thus, the present study supports the conclusion that both neurotoxic aggregates, which can form membrane pores, present this type of secondary structure in aqueous solution. In addition, the gramicidin aggregates were more stable than $A \beta$ aggregates, because the former aggregates were proteinase $K$ resistant (data not show).

\subsection{Considerations for $A \beta$ perforations and its toxic effects}

From the perforated patch clamp recordings obtained at different times, we postulate that $A \beta$ and gramicidin actions on cell membranes are fairly different. The gramicidin action appeared to be stable and selective for cations, because the $\mathrm{Cl}^{-}$gradient was not affected, providing a reversal potential near $-40 \mathrm{mV}$ for the $G A B A_{A}$ receptor-mediated current. For $A \beta$, on the other hand, using the $G A B A_{A}$-mediated reversal potential as a readout, we detected a transition state of $A \beta$ pores from cationic-selective pores to non-selective pores, suggesting that the $\mathrm{Cl}^{-}$gradient was lost during prolonged interaction of the peptide with the membrane. The data obtained with single cell imaging analysis indicate that $A \beta$ caused the entry of EtBr and 6-NBDG into the cell, providing additional support for this lack of ionic selectivity during the latter stage of membrane perforation (Fig. 7). For gramicidin pores, on the other hand, a modest entry of small fluorescent molecules (6-NBDG), but not for large fluorescent molecules $(\mathrm{EtBr})$, into the cell suggests pore sizes smaller than with $A \beta$. These data imply that, upon binding to the cell membrane, $A \beta$ may initiate its own nucleation, producing a large perforation allowing the entry of the larger molecules (Fig. 8). Interestingly, the entry of EtBr started minutes after the formation of the pore, indicating that these molecules were taken up by these $A \beta$ structures. In fact, an inhibitory peptide (NA7) that blocked these pores was also able to block the $\mathrm{EtBr}$ uptake, indicating that the $A \beta$ pores were allowing the passage of small molecules. The mechanism for this disrupting effect of $A \beta$ on neuronal membranes is largely unknown, but it is possible that the peptide interacts directly with the lipid components or by means of other neuronal proteins such as NMDA receptors (Ronicke et al., 2011, Shankar et al., 2007) or $\mathrm{Ca}^{2+}$ channels (Mezler et al., 2012).

\subsection{Neurotoxicity induced by $A \beta$ and gramicidin aggregates in hippocampal neurons}

We have recently shown that the synaptotoxicity produced by $A \beta$ is a consequence of a membrane perforation that leads to a vesicular depletion caused by a calcium-dependent mechanism (Parodi et al., 2010, Sepulveda et al., 2010). In the present study, we found that $A \beta$ aggregates can form membrane perforations, leading to a 
decrease in hippocampal neuron viability, which was blocked by reducing extracellular calcium. It is noteworthy that both aggregates decreased the presynaptic protein SV2, indicating that A $\beta$ and gramicidin aggregates produced a synaptic failure, which could be part of the early changes leading to AD (Aguayo et al., 2009). Gramicidin has been widely described as capable of producing extensive bacterial death. However, it has not been shown that gramicidin can produce neurotoxicity. Interestingly, it has recently been suggested that $A \beta$ actually possesses antimicrobial activity, and may function as an effector molecule of innate immunity in the brain (Soscia et al., 2010).

In conclusion, we demonstrate that the membrane effects of $A \beta$ have several similarities to those of gramicidin, explaining its calcium-dependent induced synaptoxicity. We postulate that the inhibition of these effects should render $A \beta$ inactive.

\section{Disclosure statement}

The authors declare that they have no conflicts of interest.

\section{Acknowledgements}

We thank Lauren Aguayo for editing the manuscript and Claudia Lopez for technical assistance. This work was supported by FONDECYT grant $N^{\circ} \underline{1100502}$ and 1060368 (L.G.A.), Ring of Research PBCT ACT-04, (L.G.A. and C.O.) and $\mathrm{CIE}-05$ grant (L.G.A.).

\section{References}

Aguayo et al., 2009. L.G. Aguayo, J. Parodi, F.J. Sepúlveda, C. Opazo. Pore-forming neurotoxin-like mechanism for Abeta oligomer-induced synaptic failure. G. Perry, R.B. Maccioni (Eds.), Current Hypotheses and Research Milestones in Alzheimer's Disease, Springer, New York (2009), pp. 13-21

Akaike, 1996. N. Akaike. Gramicidin perforated patch recording and intracellular chloride activity in excitable cells. Prog. Biophys. Mol. Biol., 65 (1996), pp. 251-264

Andersen, 1983. O.S. Andersen. Ion movement through gramicidin A channels. Interfacial polarization effects on single-channel current measurements. Biophys. J., 41 (1983), pp. 135-146

Arispe et al., 1993a. N. Arispe, H.B. Pollard, E. Rojas. Giant multilevel cation channels formed by Alzheimer disease amyloid beta-protein [A beta P-(1-40)] in bilayer membranes. Proc. Natl. Acad. Sci. U.S.A, 90 (1993), pp. 10573-10577

Arispe et al., 1993b. N. Arispe, E. Rojas, H.B. Pollard. Alzheimer disease amyloid beta protein forms calcium channels in bilayer membranes: blockade by tromethamine and aluminum. Proc. Natl. Acad. Sci. U.S.A, 90 (1993), pp. 567-571

Capone et al., 2009.

R. Capone, F.G. Quiroz, P. Prangkio, I. Saluja, A.M. Sauer, M.R. Bautista, R.S. Turner, J. Yang, M. Mayer. Amyloid-beta-induced ion flux in artificial lipid bilayers and neuronal cells: resolving a controversy. Neurotox. Res., 16 (2009), pp. 1-13

Diaz et al., 2009. J.C. Diaz, O. Simakova, K.A. Jacobson, N. Arispe, H.B. Pollard. Small molecule blockers of the Alzheimer Abeta calcium channel potently protect neurons from Abeta cytotoxicity. Proc. Natl. Acad. Sci. U.S.A, 106 (2009), pp. 3348-3353

Ebihara et al., 1995. S. Ebihara, K. Shirato, N. Harata, N. Akaike. Gramicidin-perforated patch recording: GABA response in mammalian neurones with intact intracellular chloride. J. Physiol., 484 (1995), pp. 77-86

Kawahara et al., 1997. M. Kawahara, N. Arispe, Y. Kuroda, E. Rojas. Alzheimer's disease amyloid beta-protein forms $\mathrm{Zn}(2+)$-sensitive, cation-selective channels across excised membrane patches from hypothalamic neurons. Biophys. J., 73 (1997), pp. 67-75 
Kayed et al., 2004. R. Kayed, Y. Sokolov, B. Edmonds, T.M. McIntire, S.C. Milton, J.E. Hall, C.G. Glabe.

Permeabilization of lipid bilayers is a common conformation-dependent activity of soluble amyloid oligomers in protein misfolding diseases. J. Biol. Chem., 279 (2004), pp. 46363-46366

Kelkar and Chattopadhyay, 2007. D.A. Kelkar, A. Chattopadhyay. The gramicidin ion channel: a model membrane protein. Biochim. Biophys. Acta, 1768 (2007), pp. 2011-2025

Klunk et al., 1989. W.E. Klunk, J.W. Pettegrew, D.J. Abraham. Quantitative evaluation of congo red binding to amyloid-like proteins with a beta-pleated sheet conformation. J. Histochem. Cytochem., 37 (1989), pp. $1273-1281$

Kourie, 2001. J.I. Kourie. Mechanisms of amyloid beta protein-induced modification in ion transport systems: implications for neurodegenerative diseases. Cell. Mol. Neurobiol., 21 (2001), pp. 173-213

Kourie et al., 2001. J.I. Kourie, C.L. Henry, P. Farrelly. Diversity of amyloid beta protein fragment [1-40]-formed channels. Cell. Mol. Neurobiol., 21 (2001), pp. 255-284

LeVine, 1993. $\mathrm{H}$. LeVine $3^{\text {rd }}$. Thioflavine T interaction with synthetic Alzheimer's disease beta-amyloid peptides: detection of amyloid aggregation in solution. Protein Sci., 2 (1993), pp. 404-410

Masters et al., 1985. C.L. Masters, G. Simms, N.A. Weinman, G. Multhaup, B.L. McDonald, K. Beyreuther. Amyloid plaque core protein in Alzheimer disease and Down syndrome. Proc. Natl. Acad. Sci. U.S.A, 82 (1985), pp. 4245-4249

Mezler et al., 2012. M. Mezler, S. Barghorn, H. Schoemaker, G. Gross, V. Nimmrich. A beta-amyloid oligomer directly modulates P/Q-type calcium currents in Xenopus oocytes. Br. J. Pharmacol., 165 (2012), pp. 1572-1583

Parodi et al., 2010. J. Parodi, F.J. Sepulveda, J. Roa, C. Opazo, N.C. Inestrosa, L.G. Aguayo. Beta-amyloid causes depletion of synaptic vesicles leading to neurotransmission failure. J. Biol. Chem., 285 (2010), pp. 2506-2514

Peters et al., 2013. C. Peters, E.J. Fernandez-

Perez, C.F. Burgos, M.P. Espinoza, C. Castillo, J.C. Urrutia, V.A. Streltsov, C. Opazo, L.G. Aguayo. Inhibition of amyloid beta-induced synaptotoxicity by a pentapeptide derived from the glycine zipper region of the neurotoxic peptide. Neurobiol. Aging, 34 (2013), pp. 2805-2814

Ronicke et al., 2011.

R. Ronicke, M. Mikhaylova, S. Ronicke, J. Meinhardt, U.H. Schroder, M. Fandrich, G. Reiser, M.R. Kreutz, K.G. Reymann. Early neuronal dysfunction by amyloid beta oligomers depends on activation of NR2Bcontaining NMDA receptors. Neurobiol. Aging, 32 (2011), pp. 2219-2228

Sepulveda et al., 2009. F.J. Sepulveda, C. Opazo, L.G. Aguayo. Alzheimer beta-amyloid blocks epileptiform activity in hippocampal neurons. Mol. Cell. Neurosci., 41 (2009), pp. 420-428

Sepulveda et al., 2010. F.J. Sepulveda, J. Parodi, R.W. Peoples, C. Opazo, L.G. Aguayo. Synaptotoxicity of Alzheimer beta amyloid can be explained by its membrane perforating property. PLoS One, 5 (2010), p. e11820

Shankar et al., 2007. G.M. Shankar, B.L. Bloodgood, M. Townsend, D.M. Walsh, D.J. Selkoe, B.L. Sabatini. Natural oligomers of the Alzheimer amyloid-beta protein induce reversible synapse loss by modulating an NMDA-type glutamate receptor-dependent signaling pathway. J. Neurosci., 27 (2007), pp. 2866-2875

Soscia et al., 2010.

S.J. Soscia, J.E. Kirby, K.J. Washicosky, S.M. Tucker, M. Ingelsson, B. Hyman, M.A. Burton, L.E. Goldstein, S. Duong, R.E. Tanzi, R.D. Moir. The Alzheimer's disease-associated amyloid beta-protein is an antimicrobial peptide. PLoS One, 5 (2010), p. e9505 